\title{
Ecological Protection Design Strategy for Mountain Tourism Highway Slopes
}

\author{
Zhaoyu Wang* \\ China Merchants Chongqing Communications Technology Research \& Design Institute Co., Ltd., Chongqing 400067, China

\begin{abstract}
Slope ecological protection technology is a form of slope protection with high ornamental and good economic value. At present, it is widely used in highways and urban streets' slope protection works. However, its use is not very common in mountain tourism highways; hence, it is necessary to implement strategies for mountain tourism highway slope ecological protection design to promote excellent development.
\end{abstract}

Keywords: Mountain tourism highway; Slope; Ecological protection; Design strategy

Publication date: May, 2021; Publication online: 31 May, 2021

*Corresponding author: Zhaoyu Wang, wangzhaoyu@cmhk.com

\section{Introduction}

The highway slope ecological protection in China started relatively late. There are no consistent standards in their designs; however, extensive experiences have been accumulated. In comparison to developed countries, they are relatively in a backward stage, especially pertaining mountain tourism roads, whereby research works are not thorough enough. Hence, it is necessary to conduct in-depth research and implement reasonable strategies in order to improve the quality of highway slope ecological protection and eventually, enhance the quality of mountain tourism highway slope ecological protection works.

\section{Principles of Ecology}

\subsection{Community succession and biodiversity}

The biological community belongs to a system with features of movement and change. With time, species may disappear or invade the community. Stating simply, the community and environment develop or change in corresponding directions. The main indication of community succession is the qualitative change based on the composition of the community and species. In the biodiversity principle, the ecosystem has good balance and stability with a wide range of biological species. In the case whereby an increase or decrease of one population, another population will compensate or appear inhibited to ensure good organizational function of the system. In the design of slope ecological community population, community succession must be fully considered. The development process from a primary community to an advanced community would require reasonable and scientific control works while considering the guiding maintenance in case of adverse evolution or degeneration. In the slope ecosystem, suitable species should be kept abundant. If the population is too exclusive, it is not conducive to the maintenance of the ecosystem's stability and the anti-interference ability will decline naturally. On the other hand, if the population is too large, the competition within the system will be intense. This would result in the weakening of the selforganization ability; hence, the evolution process of the plant community would be a problem. ${ }^{[1]}$

\subsection{Adaptability and limiting factors}

The principle of adaptability is that different species need specific ecological factors in the 
process of survival, growth, and reproduction. If the ecological factors are insufficient or excessive, a particular species will be limited in terms of these processes, or even forced out of the system. To be more precise, each species has a fixed range of ecological needs. Between the maximum and minimum range is the tolerance of each species, that is, the range of its adaptability.

The principle of limiting factor indicates that in the survival and reproduction of a species, basic substances must be obtained for support. A particular substance is the limiting factor when the usable amount of this substance is close to or has reached the critical value in a stable state. Hence, it is necessary to promote the regulatory capacity of the limiting factors to ensure the balance of species.

\section{Slope Ecological Protection Technology}

The ecological protection technology of slopes is based on the principle of ecology with the implementation of slope protection and land vegetation systems. In terms of the construction process, it can be divided into three components which are the design, plant construction, and management; while in terms of technology, it can also be divided into three components which are the cotton net planting belt, thick substrate spraying and continuous fiber reinforcement spraying.

\subsection{Design technology}

The design technology mainly includes two aspects: namely, vegetation design and soil design. The former uses a targeted plant species and the corresponding combination structure as its design core in regard to ecological principles. However, soil design requires effective problem-solving in terms of regulating soil nutrient, acid-base regulation, permeability improvement, and structure adjustment.

\subsection{Planting techniques}

Based on the differences in construction methods, the road vegetation construction technology can be divided into three types: artificial, mechanical, as well as artificial and mechanical composite technology. The artificial planting technology includes artificial transplanting and sowing, as well as turf paving while the mechanical construction technology includes spray seeding involving hydraulic, thick base material, guest soil, continuous fiber, and vegetation concrete. Lastly, the artificial and mechanical composite planting technology includes planting belt, geocell grass planting, three-dimensional geonet mat, rigid skeleton grass planting, concrete prefabricated skeleton grass planting, hexagonal concrete hollow block grass planting, and nutrition bag. ${ }^{[2]}$

\subsection{Nutrition technology}

There are three types of nutrition technology: namely, pruning technology, nutrient management technology, and water management technology.

\subsection{Cotton net planting belt technology}

Cotton net planting belt technology is suitable for different types of fills. Its raw material is made up of polyester fiber cotton net while the inner part is used for seed-planting and the corresponding medium ribbon complex. In construction, the planting belt is placed on the slope surface and covered with soil. The fiber net would prevent rainwater from washing off the slope surface; thus, limiting water evaporation. It also adjusts the ground temperature for conducive seed germination and growth until an organic whole constituting of roots, stems, leaves, and planting belt is formed on the slope surface. This is to achieve the effect of vegetation restoration and slope protection.

\subsection{Thick matrix spray technology}

Thick matrix spraying technology is a type of mechanical planting technology which is suitable for non-rock cutting slopes. The use of this technology is achieved by a special spray. Solid materials such as water retaining agent, microbial fertilizer, adhesive, plants substrate, seeds, and mycorrhizal fungi are sprayed onto the slope after anchoring the galvanized machine. This is to ensure 
that the substrate not only protects the slope, but also provides nutrients for the growth of plants. ${ }^{[3]}$

\subsection{Continuous fiber reinforced shotcrete technology}

Continuous fiber reinforced shotcrete technology is usually used in the ecological protection of rock slopes. It requires a special machinery to spray both, continuous fibers, and sandy soils at the same time to form interweaving materials in creating a subbase with adequate thickness. The deformation resistance and adhesion on rock slopes allow it to be reasonably used as the base; then, applying the spray coating technology of thick matrix.

\section{Ecological Protection Designs for Tourism Highway Slopes}

\subsection{Plant selections}

In the design of biological slope protection, plants should be allocated based on their own niche principle in consideration of their growth characteristics and local geographical environment. In regard to the differences in time, space, nutrition, niche, and other aspects, plants should be reasonably selected and allocated. The niche of various species should be spread out as far as possible to avoid direct competition among populations. It is beneficial to improve the green coverage per unit area. For spatial allocation, plants such as shrubs, trees, grasslands, and so on can form three-dimensional configurations. The complement of different populations may bring about mutual benefits and helps in the coordination of grounds and undergrounds. For example, the shade tolerant shrubs and positive tree species, in addition to the upper tree species and the shrubs under the forest may be utilized to create a scientifically reasonable community structure. This would not only enhance the ecological environment but also, in view of its diversity, it is conducive in promoting the growth of plants.

In terms of species selection, the focus should be on biological protection and beautification by taking into account of suitable trees, local conditions, and maintaining ecological balance.
The choice of native plants should be emphasized as they can adapt to different climates and soils. In addition to reducing workload as well as the cost of maintenance and management, it is also conducive to form a stable and growing ecological community, which may be used as a habitat for animals. Hence, this reinforces the ecological functions of the landscape itself. In regard that native plants can significantly reflect local characteristics, the diversity and ornamental value of the landscape would be improved; hence, avoiding the appearance of a uniformed landscape.

\subsection{Cultural landscape designs}

Highway landscapes have the characteristics of material forms, and their "shape" has always been the first impression for tourists. Generally speaking, "shape" only reflects the traffic functions; however, for highway landscapes, it should not only reflect that but the "meaning" as well. By integrating "shape" and "meaning," highway landscapes have more cultural connotations and artistry. Hence, in the process of designing highway landscapes, the skillful application of cultural resources is required so that it may be reasonably displayed. This would deepen the cultural connotation of the landscape environment, in addition to playing an important role in inheriting regional cultures and improving spiritual enjoyment among tourists.

In the context of slope protection design work, the regional cultural expression methods include the following points:

(1) The reasonable use of local materials. Species that show local characteristics as biological protection materials are selected.

(2) Simulation.

Slope modeling can simulate the local natural characteristics and architectural modeling, in order to display the distinctive regional characteristics and local culture.

(3) Refining.

Refining and sorting of cultural materials such as murals, sculptures, sketches, and other forms to represent national customs and human history should be emphasized, so that slope landscapes have more cultural connotations. 


\subsection{Flexible design}

For highway constructions, each project has their own distinctive characteristics. Therefore, it is necessary to fully respect the differences of each project. Based on functional and safety conditions, various technical standards need to be implemented to ensure that the design schemes are consistent with the actual needs of each project. For example, if a slope is relatively gentle, it can be designed as a curved corner to enhance its beauty and demarcate a more harmonious form with its' surrounding environment.

\section{Precautions for Slope Ecological Protection}

At present, China is still in the initial stage of implementing the slope ecological protection technology. Firstly, it is necessary to understand the essence of slope ecological protection which is to integrate the slope ecological protection of highway engineering into nature to strengthen the harmony between them. This is conducive to the implementation of the scientific development concept of highway construction. Second, in addition to the application of highway engineering related technologies, slope ecological protection also requires ample knowledge about bioecology. Hence, relevant personnel need to be educated and trained to fully understand the corresponding ecology. Third, the development of slope ecological protection works requires higher construction costs; hence, social benefits need to be improved. To promote social benefits and ensure sustainable development of slope ecology, it is not wise to blindly save costs in the construction process as long-term benefits cannot be measured only in economic terms. Fourth, in the stage of scientific research and design, the design concept of the project should be fully defined. On this basis, construction objectives should be put forward, including investments, quality, construction period, and safety objectives. At the same time, ecological benefits should be emphasized. Fifth, the application of science and technology should be strengthened, ecological construction work should be combined with local situations, and appropriate technology in the form of special research should be selected as essential supports for the smooth development of projects. Lastly, meticulous management and construction work should be carried out while abiding strictly to technical standards and specifications. Based on the actual situation, technology should be innovated and flexibly implemented to promote the harmony and unity of highway engineering, the natural environment as well as personalization. ${ }^{[4]}$

\section{Conclusion}

In view of the development and construction of mountain tourism highways, the application of slope ecological protection technology is of great significance. It is important to recognize the unity and harmony between highway and nature. The application of ecological principles does not only provide scientific theoretical guidance for construction works, but it also helps to improve the construction quality. This is to ensure that highways would resonate with the natural environment as well as to bring about a personalized and sustainable development of highway engineering.

\section{Disclosure statement}

The author declares no conflict of interest.

\section{References}

[1] Wang, H(2020). Application of ecological protection technology in highway subgrade slope protection. Gansu science and technology, 49(5): 56-58, 45.

[2]Li, H., Liu, Z(2020). Highway slope ecological protection method and its common disease analysis. Engineering technology research, 5(15): 73-74.

[3] Du, J(2020). Application of spray seeding new technology in ecological protection of expressway slope. Transportation world, (27): 18-19.

[4]Li. Y(2020). Application of ecological protection technology in mountain highway slope protection. Heilongjiang Science and technology information, (16): 106-107. 